\section{AiCS}

\title{
Acknowledgements to reviewers
}

ABCS Health Sciences acknowledges the valuable contribution from scientific reviewers in 2020:

Adriana de Oliveira França - UFMS - Campo Grande/MS

Adriana Gomes Nogueira Ferreira - UFMA - Imperatriz/MA

Adriana Torres de Lemos - UFCSPA - Porto Alegre/RS

Alan dos Santos - FMABC - Santo André/SP

Alessandra Dias de Oliveira - UFRJ - Rio de Janeiro/RJ

Alex Rey Norberto - USP - São Paulo/SP

Alex Sandro Rolland de Souza - UFPE - Recife/PE

Alexandre Delgado - IMIP - Recife/PE

Alfésio Luís Ferreira Braga - UNISANTOS - Santos/SP

Aline Ale Beraldo - USP - Ribeirão Preto/SP

Ana Amélia Camarano - IPEA - Rio de Janeiro/RJ

Ana Carolina Barco Leme - USP - São Paulo/SP

Ana Claudia Molina - UNESP - Botucatu/SP

Ana Estela Haddad - USP - São Paulo/SP

Ana Flávia Marçal Pessoa - USP - São Paulo/SP

Ana Luiza Bossolani Martins - UFMS - Campo Grande/MS

Ana Paula Amorim Moreira - UNIRIO - Rio de Janeiro/RJ

Ana Paula Guarnieri - FMABC - Santo André/SP

Ana Paula Pillatt - UNIJUI - Ijuí/RS

Ana Paula Sayuri Sato - USP - São Paulo/SP

Ana Tereza Sales - UFPB - Campina Grande/PB

Analucia de Lucena Torres - UFPE - Recife/PE

André de Camargo Smolarek - UNICENTRO - Irati/PR

Andréa Rodrigues Passos - UFBA - Salvador/BA

Andrea Wendt - UFPel - Pelotas/SC

Andréia Gonçalves Giaretta - UFSC - Florianópolis/SC

Andreia Maria Berto - FGV - São Paulo/SP

Angela Maria Alvarez - UFSC - Florianópolis/SC

Angelica Miki Stein - UNICENTRO - Guarapuava/PR

Antonio Augusto Ornellas de Souza - INCA - Rio de Janeiro/RJ

Ariana Rodrigues da Silva Carvalho - UNIOESTE - Cascavel/PR

Arthur Kaufman - UNIFESP - São Paulo/SP

Bruna Nichele da Rosa - UFRGS - Porto Alegre/RS

Camila Buonani da Silva - UNESP - Presidente Prudente/SP

Carina Fernanda Robles Angelini - UNICAMP - Campinas/SP

Carlos Eduardo Leal Vidal - FMB - Barbacena/MG

Carolina Cabral Pereira da Costa - UERJ - Rio de Janeiro/RJ

Christina Alves Peixoto - FIOCRUZ - Recife/PE 
Cintia Mesquita Correia - UFBA - Salvador/BA Claudia Nicolaevna - UFBA - Vitória da Conquista/BA Claudia Ciceri Cesa - IFSul - Sapucaia do Sul/RS Claudia Marques de Oliveira Soeiro - UEA - Manaus/AM Cristiano Queiroz de Oliveira - UCP - Petrópolis/RJ Cristina Ortiz Valete - UFSCar - São Carlos/SP Cyntia Erthal Leinig - PUC - Curitiba/PR Daniel Brito de Araujo - UFPel - Pelotas/RS Daniela Coelho Lima - UNIFAL - Alfenas/MG Daniela Siqueira Prado - UFS - Aracaju/SE Danilo Ferreira Rodrigues - UFPA - Belém/PA Dartagnan Pinto Guedes - UNOPAR - Londrina/PR Débora do Rocio Klisiowicz - UFPR - Curitiba/PR Débora Maria Befi-Lopes - USP - São Paulo/SP Débora Martins dos Santos - UERJ - Rio de Janeiro/RJ Deisiane Oliveira Souto - UFMG - Belo Horizonte/MG Diego Machado Monnerat - UNYLEYA - Rio de Janeiro/RJ Dionísia Aparecida Cusin Lamônica - USP - Bauru/SP Diorginis José Soares Ferreira - UNIVASF - Petrolina/PE Diviane Alves da Silva - UFRN - Natal/RN Edimarlei Gonsales Valério - UFRGS - Porto Alegre/RS Eduardo de Souza - UNIFESP - São Paulo/SP Einstein Francisco de Camargos - UnB - Brasília/DF Elaine Tomasi - UFPel - Pelotas/RS

Elda Lima Tavares - UERJ - Rio de Janeiro/RJ Eliana Goldfarb Cyrino - UNESP - Botucatu/SP Eliane Raquel Rieth Benetti - UFSM - Santa Maria/RS Eliany Nazaré Oliveira - UVA - Sobral/CE Elyrose Sousa Brito Rocha - UFPI - Teresina/PI Eric Bassetti Soares - Gilead Sciences - São Paulo/SP Erica Carolina Campos - UFU - Uberlândia/MG Erica Chagas Araújo - FMABC - Santo André/SP Erika Carvalho de Aquino - USP - São Paulo/SP Etna Kaliane da Silva - UFBA - Vitória da Conquista/BA Fabíola Isabel Suano de Souza - UNIFESP - São Paulo/SP Felipe Silva Neves - UFJF - Juiz de Fora/MG Fernanda Antico Benetti - FMABC - Santo André/SP Fernanda de Oliveira Souza - UEFS - Feira de Santana/BA Fernanda Ferreira Lopes - UFMA - São Luís/MA Fernanda Souza da Silva - FCMMG - Belo Horizonte/MG Flaviane Mello Lazarini - IFSC - Joinville/SC Francisco Jose Dutra Souto - UFMT - Cuiabá/MT Gabriela Rodrigues Bragagnollo - USP - Ribeirão Preto/SP Gabriela Xavier de Araujo - UFRGS - Porto Alegre/RS Gianna Fiori Marchiori - UFTM - Uberaba/MG Gláucia Regina Falsarella - UNICAMP - Campinas/SP Gláucia Virgínia de Queiroz Lins Guerra - IMIP - Recife/PE Haroldo da Silva Ferreira - UFAL - Maceió/AL Hellen Pollyanna Mantelo Cecilio - UEM - Maringá/PR Heuler Souza Andrade - UFSJ - Divinópolis/MG
Isabel Aparecida Porcatti de Walsh - UFTM - Uberaba/MG Ivone Regina Fernandes - FCMSCSP - São Paulo/SP Janderson Cleiton Aguiar - FAMERP - São José do Rio Preto/SP Januse Nogueira de Carvalho - UFCG - Campina Grande/PI João Fernando Marcolan - USP - São Paulo/SP Joice Anaize Tonon do Amaral - USP - São Paulo/SP José Carlos Rosa Pires de Souza - UEMS - Campo Grande/MS José Márcio Ribeiro - UFMG - Belo Horizonte/MG Joselina Luzia Menezes Oliveira - UFS - Aracaju/SE Júlia Dutra Rossetto - FIOCRUZ - Rio de Janeiro/RJ Karen de Souza Abrahão - INCA - Rio de Janeiro/RJ Karina Dal Sasso Mendes - USP - Ribeirão Preto/SP Karina Eiras Dela Coleta Pizzol - UNESP - Araraquara/SP Katia Fernanda Alves Moreira - UNIR - Porto Velho/RO Késia Diego Quintaes - ITAL - Campinas/SP Laércio Almeida de Melo - UFRN - Natal/RN Larissa Bueno Ferreira - UFRGS - Porto Alegre/RS Lia Silva de Castilho - UFMG - Belo Horizonte/MG Lilian Barbosa Ramos - UFBA - Salvador/BA Lucas Garcia Santana - UFVJM - Diamantina/MG Lucas Gaspar Ribeiro - USP - Ribeirão Preto/SP Lúcia Yasuko Izumi Nichiata - USP - São Paulo/SP Luciana Branco da Motta - UERJ - Rio de Janeiro/RJ Luciene Muniz Braga - UFV - Viçosa/MG Luis Fernando Barbosa Tavares - PUC - São Paulo/SP Luiza Neves - FIOCRUZ - Rio de Janeiro/RJ Marcel Nani Leite - USP - Ribeirão Preto/SP Marcela Komechen Brecailo - UNICENTRO - Guarapuava/PR Marcelo Augusto Fontenelle Ribeiro Junior - USP - São Paulo/SP Márcio Campos Oliveira - UEFS - Feira de Santana/BA Marcio Cristiano de Melo - UNICAMP - Campinas/SP Márcio Dênis Medeiros Mascarenhas - UFPI - Teresina/PI Maria Alayde Mendonça da Silva - UFAL - Maceió/AL Maria Alix Leite Araujo - UNIFOR - Fortaleza/CE Maria Antonieta Spinoso Prado - USP - Ribeirão Preto/SP Maria das Graças Monte Mello Taveira - UFAL - Maceió/AL Maria de Fátima de Souza - UFRN - Natal/RN

Maria do Perpétuo Socorro de Sousa Nóbrega - USP - Ribeirão Preto/SP

Maria do Socorro Cavalcante - UFC - Fortaleza/CE Maria Goretti da Cunha Lisboa - UEPB - Campina Grande/PB Maria Isabel Ramos do Amaral - UNICAMP - Campinas/SP Maria Ivoneide Veríssimo de Oliveira - UFC - Fortaleza/CE Maria Rosilene Cândido Moreira - UFCA - Juazeiro do Norte/CE Mariana André Honorato Franzoi - UnB - Brasília/DF Mateus Camaroti Laterza - UFJF - Juiz de Fora/MG Max Moura de Oliveira - UnB - Brasília/DF Maysa Venturoso Gongora Buckeridge Serra - UNIFRAN - Franca/SP

Meiry Fernanda Pinto Okuno - UNIFESP - São Paulo/SP 
Mercedes Trentini - UFSC - Florianópolis/SC

Mirelle de Oliveira Saes - UFPel - Pelotas/RS

Miriam Ventura da Silva - UFRJ - Rio de Janeiro/RJ

Mônica Leila Portela de Santana - UFBA - Salvador/BA

Monique Binotto - HCPA - Porto Alegre/RS

Natália da Silva Freitas Marques - UFAC - Rio Branco/AC

Natalia Pires de Vasconcelos - INSPER - São Paulo/SP

Neilton Araujo de Oliveira - UFT - Palmas/TO

Nivaldo Antonio Parizotto - UFSCar - São Carlos/SP

Olagide Wagner de Castro - UFAL - Maceió/AL

Oscar Cesar Pires - UNITAU - Taubaté/SP

Osmar Clayton Person - UNIFESP - São Paulo/SP

Patrícia Fernanda Roesler Bertolini - PUC - Campinas/SP

Patrícia Treviso - UFRGS - Porto Alegre/RS

Paulo Diniz da Gama - PUC - Sorocaba/SP

Paulo do Carmo Fonseca - UFMG - Belo Horizonte/MG

Paulo Henrique Ferreira Bertolucci - UNIFESP - São Paulo/SP

Paulo Roberto Lacerda Leal - UFC - Fortaleza/CE

Pedro Marco Karan Barbosa - FAMEMA - Marília/SP

Rafael Aiello Bomfim - UFMS - Campo Grande/MS

Rafael Haeffner - USP - São Paulo/SP

Rafael Silva Marconato - UNICAMP - Campinas/SP

Rafaela Lira Formiga Cavalcanti de Lima - UFPB - João Pessoa/PB

Raquel Agnelli Mesquita-Ferrari - UNINOVE - São Paulo/SP

Raquel Saccani - UCS - Caxias do Sul/RS

Renata Elizabete Pagotti da Fonseca - UFSCar - São Carlos/SP

Renata Macedo Martins Pimentel - FMABC - Santo André/SP

Ricardo Luiz Dantas Machado - UFF - Niterói/RJ

Ricardo Porto Tedesco - UNICAMP - Campinas/SP

Rita de Cássia Melão de Morais - UnB - Brasília/DF

Rita Leal Sperotto - UNICRUZ - Cruz Alta/RS

Roberta Ferrari Marback - UFBA - Salvador/BA

Rodrigo Caetano Arantes - ESP - Belo Horizonte/MG
Rodrigo Dalke Meucci - FURG - Rio Grande/RS

Rodrigo Marques Tonella - UNICAMP - Campinas/SP

Rosa Gomes dos Santos Ferreira - UFRJ - Rio de Janeiro/RJ

Rosa Maria Graziano - USP - São Paulo/SP

Rosa Maria Soares Madeira Domingues - FIOCRUZ -Rio de Janeiro/RJ

Rosana Amora Ascari - UDESC - Chapecó/SC

Rosilane de Lima Brito Magalhães - UFPI - Teresina/PI

Rúbia Formighieri Giordani - UFPR - Curitiba/PR

Sabrina da Silva de Souza - UFSC - Florianópolis/SC

Selma Denis Squassoni - FMABC - Santo André/SP

Selma Maria da Fonseca Viegas - UFSJ - São João del Rei/MG

Sergio Pedro Baldassin - FMABC - Santo André/SP

Sheila Jacques Oppitz - UFSM - Santa Maria/RS

Silvia Helena Arias Bahia - UFPA - Belém/PA

Sônia Beatriz Cocaro de Souza - UFRGS - Porto Alegre/RS

Susana Cararo Confortin - UFSC - Florianópolis/SC

Tamires Aparecida Bitencourt - UNAERP - Ribeirão

Preto/SP

Tânia Rosane Bertoldo Benedetti - UFSC - Florianópolis/SC

Terezinha Inez Estivalet Svidzinski - UEM - Maringá/PR

Thaisy Cristina Honorato Santos Alves - UFBA - Salvador/BA

Thereza Maria Magalhães Moreira - UECE - Fortaleza/CE

Thiago Fernandes - USP - São Paulo/SP

Thomaz Bittencourt Couto - HIAE - São Paulo/SP

Vander Monteiro da Conceição - UFFS - Chapecó/SC

Vânia Tie Koga Ferreira - UNIFAP - Macapá/AP

Veronica Silva Vilela - UERJ - Rio de Janeiro/RJ

Vilma Beltrame - PUCRS - Porto Alegre/RS

Vinicius Cappo Bianco - UNIARARAS - Araras/SP

Vitor Tigre Martins Rocha - UFMG - Belo Horizonte/MG

Vládia Jamile dos Santos Jucá - UFC - Fortaleza/CE

Wanderley Matos Reis Júnior - UESB - Jequié/BA

Zuleyce Maria Lessa Pacheco - UFJF - Juiz de Fora/MG 